\title{
Analytical validation of a psychiatric pharmacogenomic test
}

\author{
Michael R Jablonski ${ }^{1}$, Nina King ${ }^{1}$, Yongbao Wang ${ }^{1}$, Joel G Winner ${ }^{1}$, Lucas R Watterson ${ }^{1}$, \\ Sandra Gunselman ${ }^{1}$ \& Bryan M Dechairo*,1 \\ ${ }^{1}$ Assurex Health, Inc., Mason, OH, USA \\ *Author for correspondence: Tel.: +1 801584 1134; bdechairo@assurexhealth.com
}

\begin{abstract}
Aim: The aim of this study was to validate the analytical performance of a combinatorial pharmacogenomics test designed to aid in the appropriate medication selection for neuropsychiatric conditions. Materials \& methods: Genomic DNA was isolated from buccal swabs. Twelve genes (65 variants/alleles) associated with psychotropic medication metabolism, side effects, and mechanisms of actions were evaluated by bead array, MALDI-TOF mass spectrometry, and/or capillary electrophoresis methods (GeneSight Psychotropic, Assurex Health, Inc.). Results: The combinatorial pharmacogenomics test has a dynamic range of 2.5-20 ng/ $\mu$ l of input genomic DNA, with comparable performance for all assays included in the test. Both the precision and accuracy of the test were $>99.9 \%$, with individual gene components between 99.4 and $100 \%$. Conclusion: This study demonstrates that the combinatorial pharmacogenomics test is robust and reproducible, making it suitable for clinical use.
\end{abstract}

First draft submitted: 18 December 2017; Accepted for publication: 18 January 2018; Published online: 7 February 2018

Keywords: analytical validation $\bullet$ combinatorial pharmacogenomics $\bullet$ genetic testing $\bullet$ neuropsychiatric conditions - pharmacogenomics

Pharmacogenomics is a burgeoning branch of personalized medicine that has emerged from the need to minimize the cumbersome trial and error approach to identify safe and effective drug treatment regimens for patients with mental illness. Fewer than half of the patients respond to their initial antidepressant treatment and response rates decline even further with additional treatment trials [1,2]. However, pharmacogenomic molecular testing can improve medication selection by evaluating genetic variations known to affect the physiological effects of some psychiatric medications. For example, many psychiatric medications are metabolized by one or more cytochrome P450 (CYP) enzymes. As such, genetic variations that impact the enzymatic activity of CYPs will have a direct impact on the metabolism, and therefore, safety and efficacy of relevant drugs. Given these known gene-drug interactions, the US FDA now makes genotype-specific psychiatric medication recommendations, including modified dose recommendations [3]. For example, the FDA recommends that the maximum dose of Celexa (citalopram) be decreased from 40 to $20 \mathrm{mg}$ /day for 'CYP2C19 poor metabolizers' due to a substantial increase in the peak plasma concentration and area under the plasma concentration time curve for individuals with this genotype [4].

Understanding an individual's genomic variation can be used to guide medication selection with the goal of decreasing adverse drug events and increasing efficacy. However, many psychiatric drugs are metabolized by multiple pathways and act on multiple targets. As such, genetic variations in many different genes may impact the pharmacokinetics (rate of metabolism) as well as the pharmacodynamics (mechanism of action) of specific medications. A number of genetic variants are also associated with the increased risk for adverse drug reactions [5]. For example, the presence of certain HLA alleles can contribute to an increased risk for carbamazepine-induced cutaneous adverse drug reactions [6].

In response to these clinical concerns, a combinatorial pharmacogenomic test has been developed to guide psychiatric medication selection (GeneSight Psychotropic) [7]. This test detects genetic variations in several genes associated with the pharmacokinetics, pharmacodynamics, and treatment related side effects for medications that are used to treat neuropsychiatric disorders. The genotype of each component is used to generate a weighted combinatorial report in order to rank medications according to the severity of gene-drug interactions [7]. Previous studies 


\begin{tabular}{|c|c|c|c|c|}
\hline \multirow[t]{2}{*}{ Gene } & \multirow[t]{2}{*}{ Alleles/variants evaluated } & \multicolumn{3}{|c|}{ Assays used in genotyping } \\
\hline & & Bead array & MALDI-TOF & $\begin{array}{l}\text { PCR + capillary } \\
\text { electrophoresis }\end{array}$ \\
\hline CYP1A2 & $\begin{array}{l}-3860 \mathrm{G}>\mathrm{A},-2467 \mathrm{~T}>\text { delT, }-739 \mathrm{~T}>\mathrm{G},-729 \mathrm{C}>\mathrm{T},-163 \mathrm{C}>\mathrm{A}, 125 \mathrm{C}>\mathrm{G}, \\
558 \mathrm{C}>\mathrm{A}, 2116 \mathrm{G}>\mathrm{A}, 2473 \mathrm{G}>\mathrm{A}, 2499 \mathrm{~A}>\mathrm{T}, 3497 \mathrm{G}>\mathrm{A}, 3533 \mathrm{G}>\mathrm{A} \\
5090 \mathrm{C}>\mathrm{T}, 5166 \mathrm{G}>\mathrm{A}, 5347 \mathrm{C}>\mathrm{T}\end{array}$ & $x$ & $x$ & \\
\hline CYP2B6 & $* 4, * 6, * 9$ & $x$ & $x$ & \\
\hline CYP2C9 & $* 2, * 3, * 4, * 5, * 6$ & $x$ & $x$ & \\
\hline CYP2C19 & $* 2, * 3, * 4, * 5, * 6, * 7, * 8, * 17$ & $x$ & $x$ & \\
\hline CYP2D6 & $\begin{array}{l}* 2, * 2 A, * 3, * 4, * 5 \text { (gene deletion), *6, *7,*8,*9,*10,*11,*12, } \\
* 14, * 15, * 17, * 41 \text {, gene duplication }\end{array}$ & $x$ & $x$ & $\mathbf{X}^{\dagger}$ \\
\hline CYP3A4 & $* 13, * 15 \mathrm{~A}, * 22$ & $x$ & $x$ & \\
\hline UGT1A4 & $* 3$ & $x$ & $x$ & \\
\hline UGT2B15 & $* 2$ & $x$ & $x$ & \\
\hline HTR2A & $-1438 G>A$ & $x$ & $x$ & \\
\hline SLC6A4 (rs4795541) & $\mathrm{L}, \mathrm{S}$ & & & $x$ \\
\hline HLA-A & $\begin{array}{l}\text { rs1061235 polymorphism indicating presence of the HLA-A*3101 } \\
\text { allele or certain HLA-A*33 alleles }\end{array}$ & $x$ & $x$ & \\
\hline HLA-B & $\begin{array}{l}\text { HLA-B*1502 and closely related HLA-B alleles *1511, *1513 and } \\
* 1515\end{array}$ & $x$ & & $X^{\ddagger}$ \\
\hline \multicolumn{5}{|c|}{$\begin{array}{l}\text { The alleles and/or genetic variants tested in each gene are shown along with the assays utilized to assess genotype. } \\
{ }^{\dagger} \text { CYP2D } 6 \text { duplication and deletion only. } \\
\ddagger \text { HLA-B* } 1502 \text { and SLC6A4/HLA-B* } 1502 \text { multiplex assay. }\end{array}$} \\
\hline
\end{tabular}

have demonstrated that this combinatorial pharmacogenomic test predicts response to psychiatric medications, with a superior performance relative to single-gene analysis [8]. In addition, utilization of this test has been shown to improve patient response to selected medications $[9,10]$ and decrease overall healthcare costs $[11,12]$.

The analytical performance of molecular tests is an important component of appropriate clinical use, as the ability to detect the presence or absence of a particular biomarker, gene or genetic variant must be robust and reproducible. For many molecular tests, evaluating the analytical performance is fairly straightforward and is based on dichotomous (i.e., positive vs negative) results. However, many pharmacokinetic and pharmacodynamic markers are highly variable and result in genotypes and genotype combination(s) that produce a range of phenotypes. For example, metabolizer phenotypes of CYP enzymes can range from 'poor metabolizer' to 'ultra-rapid metabolizer' phenotypes. Therefore, pharmacogenomic tests must take into consideration both the analytical performance of the assay (precision and accuracy) as well as the ability of tested markers to affect a phenotype (clinical relevance).

Despite the large number of commercially available pharmacogenomic tests, very few analytical validations have been published for pharmacogenomic testing. A recent study by Bousman et al. highlighted a high degree of variability even in the recommendations provided by commercially available pharmacogenetic tests [13], despite recommendations from the Clinical Pharmacogenetics Implementation Consortium [14]. In light of the clinical importance of validating the performance of pharmacogenomic tests, we present the analytical validation of a combinatorial pharmacogenomic test with regards to the individual gene components as well as the genotype results.

\section{Materials \& methods}

Pharmacogenomic testing

The combinatorial pharmacogenomic test (GeneSight Psychotropic, Assurex Health, OH, USA) included 65 alleles and variants across 12 genes (Table 1): CYP1A2 (15 alleles), CYP2B6 (4 alleles), CYP2C9 (6 alleles), CYP2C19 (9 alleles), CYP2D6 (17 alleles and duplication), CYP3A4 (4 alleles), UGT1A4 (2 alleles), UGT2B15 (2 alleles), HTR2A ( 2 alleles), the long and short 5HTTLPR variants of the SLC6A4 serotonin transporter gene (2 alleles), HLA-A (*3101 associated SNP rs1061235) and HLA-B (1 allele).

This test has been validated using genomic DNA. Samples utilized here were submitted for clinical testing (Assurex Health) unless specified otherwise. Genomic DNA was collected using buccal swab samples as previously described [8]. This study utilized de-identified data collected in the course of normal healthcare operations. 
Combinatorial pharmacogenomic testing involves two main components - evaluating the genotype of each variant/allele and interpreting the collective genotypes. The genotype for each allele/variant was determined using laboratory-developed methods that utilized both manufacturer-designed and custom-designed primers. Analysis of HLA-B*1502 and SLC6A4 was completed by capillary electrophoresis of PCR products using laboratory-developed multiplex assays. Analysis of CYP1A2, CYP2B6, CYP2C19, CYP2C9, CYP3A4, HTR2A, HLA-A*3101, UGT1A4 and UGT2B15 variants was completed by either multiplex iPLEX MassARRAY MALDI-TOF mass-spectrometry assays (Agena Bioscience, CA, USA) or multiplex Luminex xTAG bead array assays (Luminex Corporation, TX, USA). CYP2D6 genotype was determined using either iPLEX MassARRAY assays in combination with the laboratory-developed long-range PCR assays for the CYP2D6 deletion and duplication or using commercially available xTAG CYP2D6 v3 assay from Luminex Corporation [15]. A list of the tested genetic variants and the analysis methods is presented in Table 1, with additional details described in the Supplementary Material.

All the assays were performed in batches that contained genomic DNA sample(s) from Coriell Institute for Medical Research with known genotypes as positive control sample(s), a negative control sample (nuclease-free water) and/or an extraction control sample (a negative control sample that underwent DNA extraction process alongside the patient samples). Batch results were considered passing if the positive control sample(s) displayed the expected genotype and negative/extraction control samples did not produce any signal(s) above the established thresholds. To be considered passing results, samples were also required to meet prespecified criteria for each assay as described in the Supplementary Material.

For passing samples, the genotypes for each measured allele/variant were evaluated by a pharmacogenomic algorithm [7]. This algorithm analyzed 12 different genes to weigh their combined influence on patient response to 55 different psychotropic medications. Based on this analysis, medications were placed into one of three advisory categories based on the pharmacokinetic and pharmacodynamic implications: 'use as directed', 'moderate gene-drug interaction' and 'significant gene-drug interaction'.

\section{Dynamic range \& detection limit}

The range of input DNA that produces reproducible test results was evaluated using a dilution series of samples with final DNA concentrations ranging from 1 to $100 \mathrm{ng} / \mu \mathrm{l}$ (DNA input amounts from 5 to $500 \mathrm{ng}$ ). Genomic DNA samples were obtained from the National Institute of General Medical Sciences (NIGMS) Human Genetic Cell Repository at the Coriell Institute for Medical Research (repository IDs listed in Supplementary Table 1) and diluted to the desired concentration. Samples that contained genetic variants included in the pharmacogenomic panel were selected for use in this analysis. Each concentration was tested in triplicate on each assay for a total of 24 samples per concentration. The range of concentrations at which passing results were consistently obtained on all the assays was determined.

\section{Precision}

The precision of the combinatorial pharmacogenomic test was evaluated via repeatability (i.e., closeness of agreement between independent test results for the same samples and under the same conditions) and reproducibility (i.e., closeness of agreement between independent test results for the same measure and under different conditions). Repeatability was assessed by testing unique samples in triplicate by the same technician and same instrument on different days. Reproducibility was assessed by comparing the results produced by different technicians and different instruments.

The precision of all assays was evaluated using eight unique batches tested in triplicate, with the exception of the electrophoresis assay for the CYP2D6 deletion. The bead array assays were evaluated using batches of 10 samples (CYP1A2, CYP2B6, CYP2C9, CYP3A4, HTR2A) or 14 samples (HTR2A, HLA-A*3101, HLA-B*1502, UGT1A4, UGT2B15) for a total of 240 or 336 samples, respectively. The MALDI-TOF assay was evaluated using batches of seven for a total of 168 samples. The electrophoresis assays for SLC6A4 and HLA-B were tested using batches of 31 samples for a total of 744 samples. The electrophoresis assay for the CYP2D6 duplication was tested using batches of ten samples (120 total samples). The electrophoresis assay for the CYP2D6 deletion was tested using four batches of ten samples ( 40 total samples).

\section{Accuracy}

The accuracy of the combinatorial pharmacogenomic test was evaluated using samples with known genotypes. For CYP1A2, CYP2B6, CYP2C19, CYP2C9, CYP2D6 and CYP3A4, accuracy was evaluated using reference 
Table 2. Dynamic range assessment.

\begin{tabular}{|c|c|c|c|c|c|c|}
\hline \multirow{2}{*}{$\begin{array}{l}\text { DNA concentration } \\
(\mathrm{ng} / \mu \mathrm{l})\end{array}$} & \multirow[t]{2}{*}{ Bead array assays } & \multirow[t]{2}{*}{ MALDI-TOF assay } & \multicolumn{4}{|c|}{ PCR + capillary electrophoresis assays } \\
\hline & & & SLC6A4 & SLC6A4/HLA-B*1502 & CYP2D6 duplication & CYP2D6 deletion \\
\hline $60-100$ & $18 / 18$ & $3 / 4$ & $\mathrm{n} / \mathrm{a}$ & $6 / 6$ & $6 / 6$ & $6 / 6$ \\
\hline $40-50$ & $18 / 18$ & $3 / 4$ & $9 / 9$ & $6 / 6$ & $6 / 6$ & $6 / 6$ \\
\hline 20 & $18 / 18$ & $4 / 4$ & $9 / 9$ & $6 / 6$ & $6 / 6$ & $6 / 6$ \\
\hline 10 & $18 / 18$ & $4 / 4$ & $\mathrm{n} / \mathrm{a}$ & $6 / 6$ & $6 / 6$ & $6 / 6$ \\
\hline 5 & $18 / 18$ & $4 / 4$ & $\mathrm{n} / \mathrm{a}$ & $6 / 6$ & $6 / 6$ & $6 / 6$ \\
\hline 2.5 & $18 / 18$ & $4 / 4$ & $9 / 9$ & $6 / 6$ & $6 / 6$ & $6 / 6$ \\
\hline 1 & $18 / 18$ & $4 / 4$ & $\mathrm{n} / \mathrm{a}$ & $6 / 6$ & $6 / 6$ & $5 / 6$ \\
\hline
\end{tabular}

samples from the NIGMS Human Genetic Cell Repository at the Coriell Institute for Medical Research (repository IDs listed in the Supplementary Table 1). These reference samples contained mutations of clinical importance that have been confirmed by multiple volunteer laboratories using different testing platforms [16]. For UGT1A4, UGT2B15, HLA-A*3101 and HLA-B*1502, accuracy was also evaluated using genomic DNA samples from NIGMS Human Genetic Cell Repository at the Coriell Institute for Medical Research for samples included in the 1000 Genomes Project. For variants in these genes, expected genotypes were derived from the 1000 Genomes Project [17]. Additionally, proficiency-testing samples from the College of American Pathologists (CAP) [18] were used as reference samples for CYP2C19, CYP2C9 and CYP2D6 genotypes. Results of proficiency sample testing were submitted to the CAP and compared with the expected results and results submitted by other laboratories.

The test accuracy was evaluated using additional methods when available. Polymorphisms in CYP1A2, CYP2B6, HTR2A, UGT1A4 and UGT2B15 were confirmed by an orthogonal independent test method performed internally or by an independent laboratory. This included Sanger sequencing for CYP1A2 and HTR2A, PCR followed by restriction fragment length polymorphism analysis for CYP2B6, pyrosequencing for UGT1A4 and qPCR assay for UGT2B15. Since no reference samples were available for SLC6A4 'S' and 'L' polymorphism, the results obtained from Assurex Health Laboratories for ten samples were confirmed by two independent laboratories: Cincinnati Children's Hospital Medical Center Molecular Diagnostic Laboratory (OH, USA) or PGX Laboratories (KY, USA).

Only two and three reference samples were available for HLA-A*3101 and HLA-B*1502, respectively. Therefore, four to seven positive and negative patient samples were tested and the results were confirmed using high-resolution HLA sequence-based typing performed by Histogenetics (NY, USA) and an alternative PCR method using the HLA-A*3101 detection kit (Pharmigene, Inc., CA, USA), or the HLA-B*1502 detection kit (Pharmigene, Inc).

\section{Allele frequencies}

To evaluate assay performance, the minor allele frequencies (MAFs) for each SNP included in combinatorial pharmacogenomics test were evaluated among a clinical testing population that represents admixed North American population. The observed allele frequencies for different testing methods were obtained by direct counting and compared with MAFs published in the National Center for Biotechnology Information (NCBI) SNP database (dbSNP) (1000 Genomes Project: Eastern Asian, $\mathrm{n}=504$; African, $\mathrm{n}=661$; European, $\mathrm{n}=503$; admixed American, $\mathrm{n}=347$; South Asian, $\mathrm{n}=489$; and the Exome Aggregation Consortium Aggregated Populations, $\mathrm{n}=60,706)$ [19]

\section{Results}

\section{Dynamic range}

The range of genomic DNA concentrations that will produce reliable combinatorial pharmacogenomic test results was determined. The bead array assays and electrophoresis assays for SLC6A4/HLA-B*1502 PCR and the CYP2D6 duplication produced passing results for all samples over the full range of DNA concentrations evaluated (1$100 \mathrm{ng} / \mu \mathrm{l}$ ) (Table 2). The SLC6A4 PCR and electrophoresis assays produced passing results for all evaluated DNA concentrations $(2.5,20,40-50 \mathrm{ng} / \mu \mathrm{l})$. The MALDI-TOF assay had a $25 \%$ pass rate for samples between 40 and $100 \mathrm{ng} / \mu \mathrm{l}$; however, all the samples with 1-20 ng/ $\mu \mathrm{l}$ of input DNA produced passing results. The long-range PCR and electrophoresis assays for the CYP2D6 deletion had an 83\% pass rate at a DNA concentration of $1 \mathrm{ng} / \mu \mathrm{l}$. Thus, the dynamic range of all the assays was $2.5-20 \mathrm{ng} / \mu \mathrm{l}$ of genomic DNA. 


\begin{tabular}{|c|c|c|c|c|}
\hline Assay & Gene/variant & Samples evaluated & Repeatability & Reproducibility \\
\hline \multirow[t]{9}{*}{ Bead array } & CYP1A2 & 240 & $100 \%$ & $100 \%$ \\
\hline & CYP2C9 & 240 & $100 \%$ & $100 \%$ \\
\hline & CYP2C19 & 240 & $100 \%$ & $100 \%$ \\
\hline & CYP3A4 & 240 & $100 \%$ & $100 \%$ \\
\hline & HTR2A & 240 & $100 \%$ & $100 \%$ \\
\hline & HLA-A*3101 & 336 & $100 \%$ & $100 \%$ \\
\hline & HLA-B*1502 & 336 & $100 \%$ & $100 \%$ \\
\hline & UGT1A4 & 336 & $99 \%$ & $99 \%$ \\
\hline & UGT2B15 & 336 & $100 \%$ & $100 \%$ \\
\hline \multirow{7}{*}{ MALDI-TOF } & CYP2C19 & 168 & $100 \%$ & $100 \%$ \\
\hline & CYP2D6 & 168 & $100 \%$ & $100 \%$ \\
\hline & CYP3А4 & 168 & $100 \%$ & $100 \%$ \\
\hline & HTR2A & 168 & $>99.9 \%$ & $>99.9 \%$ \\
\hline & HLA-A*3101 & 168 & $100 \%$ & $100 \%$ \\
\hline & UGT1A4 & 168 & $99 \%$ & $99 \%$ \\
\hline & UGT2B15 & 168 & $100 \%$ & $100 \%$ \\
\hline \multirow{3}{*}{$\begin{array}{l}\text { PCR + capillary electrophoresis } \\
\text { assays }\end{array}$} & HLA-B*1502 & 744 & $100 \%$ & $100 \%$ \\
\hline & SLC6A4 & 744 & $100 \%$ & $100 \%$ \\
\hline & CYP2D6 duplication & 120 & $100 \%$ & $100 \%$ \\
\hline
\end{tabular}

\section{Precision}

The precision of each testing method was assessed by comparing genotype results for samples tested under the same conditions (repeatability) and under different conditions (reproducibility). Samples tested under the same conditions were $\geq 99.9 \%$ concordant for all assays, with the exception of the bead array assay for UGT1A4 *3 polymorphism (Table 3). Similarly, samples tested under different conditions were $\geq 99.9 \%$ concordant for all assays other than the UGT1A4 bead array assay (Table 3). Discordant UGT1A4 genotypes were observed for one replicate of the same sample to produce a repeatability and reproducibility of $99 \%$. This was due to low median fluorescence intensity signals leading to abnormal analyte ratios.

\section{Accuracy}

The accuracy of the combinatorial pharmacogenomic test was evaluated by assessing samples with known genotypes via at least two independent methods. The methods of genotype confirmation are described in detail in Supplementary Table 2. For CYP1A2, CYP2B6, CYP2C19, CYP2C9, CYP2D6, CYP3A4, HTR2A, UGT1A4, UGT2B15 and SLC6A4, the overall concordance between laboratory-determined genotypes and the expected genotypes obtained using a reference method was 100\% (Table 4).

The presence or absence of the HLA-A*3101 allele was indirectly evaluated using the rs1061235A $>$ T SNP, which is in linkage disequilibrium $\left(\mathrm{r}^{2}=1\right)$ with HLA-A*3101 in European populations [20]. All the samples containing the HLA-A*3101 allele were positive for rs1061235A>T variant; however, some samples that were positive for this variant contained HLA-A*3301. Overall, 67\% (8/12) of rs $1061235 \mathrm{~A}>\mathrm{T}$ positive samples were HLA-A*3101 positive and $33 \%(4 / 12)$ were HLA-A*3301 positive. This is consistent with a previous study, which demonstrated that this SNP is associated with the presence of HLA-A*33 alleles [21]. As both HLA-A *3101 and HLA-A*3301 alleles are associated with rs1061235 A > T variant, this analysis was $100 \%$ concordant with the expected genotypes. 
Table 4. Accuracy of assay genotyping.

\begin{tabular}{|c|c|c|}
\hline Gene & Concordance with reference method 1 & Concordance with reference method $2^{\dagger}$ \\
\hline \multicolumn{3}{|c|}{ Primary method of genotype confirmation: CDC GeT-RM } \\
\hline CYP1A2 & $100 \%(n=6)$ & $100 \%(n=10)$ \\
\hline CYP2B6 & $100 \%(n=11)$ & $100 \%(n=125)$ \\
\hline CYP2C19 & $100 \%(n=12)$ & $100 \%(n=39)$ \\
\hline CYP2C9 & $100 \%(n=22)$ & $100 \%(n=39)$ \\
\hline CYP2D6 & $100 \%(n=22)$ & $100 \%(n=39)$ \\
\hline CYP3A4 & $100 \%(n=11)$ & \\
\hline \multicolumn{3}{|c|}{ Primary method of genotype confirmation: 1000 Genomes Project } \\
\hline UGT1A4 & $100 \%(n=3)$ & $100 \%(n=21)$ \\
\hline UGT2B15 & $100 \%(n=3)$ & $100 \%(n=47)$ \\
\hline rs1061235 (T) & $100 \% \ddagger(n=12)$ & $100 \%(n=12)$ \\
\hline HLA-B*1502 & $100 \%(n=2)$ & $83 \%(n=6)$ \\
\hline \multicolumn{3}{|c|}{ Primary method of genotype confirmation: CCHMC Molecular Diagnostics Lab } \\
\hline HTR2A & $100 \%(n=24)$ & $100 \%(n=10)$ \\
\hline SLC6A4 & $100 \%(n=10)$ & $100 \%(n=10)$ \\
\hline \multicolumn{3}{|c|}{$\begin{array}{l}\text { Concordance between laboratory-determined genotypes and genotypes determined by the different reference method(s). } \\
\dagger \text { Includes the evaluation of CAP proficiency samples, testing by an independent laboratory or in-house testing by orthogonal methods. } \\
\text { ¥Includes the detection of either HLA-A*3101 or HLA-A*3301. } \\
\text { CAP: College of American Pathologists; CCHMC: Cincinnati Children's Hospital Medical Center; CDC GeT-RM: Centers for Disease Control Genetic testing reference materials coordination } \\
\text { program. }\end{array}$} \\
\hline
\end{tabular}

For HLA-B*1502, one false positive result was produced for the reference samples, for a concordance of $83 \%$ (Table 4). This is consistent with a separate analysis of a large patient cohort, which showed that other closely related HLA-B*15 alleles (e.g., ${ }^{*} 1515,{ }^{*} 1513$ and ${ }^{*} 1511$ ) may be also detected by the HLA-B*1502 PCR assay to yield some false positive results (data not shown).

Overall, the laboratory determined genotypes were determined in 496 reference samples. With only one false positive result, the overall accuracy of the combinatorial pharmacogenomic test was $99.8 \%$.

\section{Allele frequencies}

Observed allele frequencies can be used to monitor assay performance both internally and in comparison to published genotype frequencies. As shown in Supplementary Table 3, MAFs observed in patients representing an admixed North American population who underwent clinical testing using different methods were in agreement with the expected allele frequencies for each other. This included the detection of all the tested genetic variants, including rare variants.

\section{Discussion}

Consideration of pharmacogenomic information can be important in appropriate medication selection for neuropsychiatric conditions, especially in individuals with extreme phenotypes. Much like information about drug-drug interactions, pharmacogenomics can be a helpful adjunct to clinical decision-making. The ability of combinatorial pharmacogenomic testing to predict outcomes (an important measure of clinical validity) has been demonstrated in several studies $[7,10,12,22]$. Improvement in outcomes using combinatorial pharmacogenomic testing (clinical utility) has also been tested [7,10-11,22]. In order to ensure appropriate patient care, it is also important to show that the test findings are an accurate representation of a patient's genotype. Here, we report the analytic validity of all the genes and genetic variants tested as part of a 12-gene combinatorial pharmacogenomic test.

The data presented here show that all assays produced passing results for samples with input DNA concentrations between 2.5 and $20 \mathrm{ng} / \mu \mathrm{l}$. The concentration of genomic DNA obtained from buccal swabs varies greatly, ranging from 2 to $72 \mathrm{ng} / \mu \mathrm{l}$ or even higher. As such, samples with a concentration of $>20 \mathrm{ng} / \mu \mathrm{l}$ are diluted for testing. However, with a lower limit of $2.5 \mathrm{ng} / \mu \mathrm{l}$, the DNA obtained from buccal swabs is sufficient for reliable testing.

The repeatability and reproducibility was $\geq 99.9 \%$ for all individual genes, with the exception of UGT1A4. For this gene, low fluorescence intensity in one sample resulted in discordant findings. The data analysis parameters for UGT1A4 were subsequently adjusted to identify and re-queue samples with a similar issue, thereby minimizing 
the risk of reporting incorrect results. Still, the overall precision of the pharmacogenomic test was $>99.9 \%$. This shows that the laboratory testing methods are robust and reproducible under all conditions.

The accuracy of the genotypes determined by the combinatorial pharmacogenomic test was evaluated for all tested genes. This was done using reference samples with known genotypes when available. Test results were $100 \%$ concordant for all genes evaluated against reference samples with known genotypes. Test results were also $100 \%$ concordant with the genotypes for clinical samples obtained by independent or orthogonal testing for all variants and alleles with the exception of HLA-B* 1502 , for which there was one false positive. Overall, the accuracy of genotype results produced by this combinatorial pharmacogenomic test was $99.8 \%$. The false positive result for HLA-B* 1502 was likely due to closely related alleles with high DNA and protein sequence homology. While these related alleles are rare, additional studies are needed to investigate any possible role they may play in drug hypersensitivity reactions.

In an admixed North American population, the observed allele frequencies measured using the bead array assays were comparable to those measured using MALDI-TOF assays (or PCR/capillary electrophoresis-based assay for HLA-B*1502). This indicates that the test performance is comparable between methods that utilize different primer sets and analytical platforms. The allele frequencies observed using the combinatorial pharmacogenomic testing methods were also compared with those reported in the NCBI dbSNPs. Overall, allele frequencies published in NCBI dbSNPs show great variability in different populations. For example, allele frequencies for the CYP2D6100C > T (rs1065852) genetic variant ranged from 11\% in an African population to $57 \%$ in an East Asian population. The allele frequencies observed in the cohort of patients who had clinical testing here were most similar to the European population data; however, clinical testing populations may have different allele distributions due to enrichments or depletions consistent with the nature of the genetic condition being evaluated.

\section{Conclusion}

The current framework for the evaluation of pharmacogenomic tests is based on the standards established for molecular diagnostic tests. For most pharmacogenomic biomarkers, it is not always clear which factors should be evaluated for sensitivity and specificity, as these could include enzyme activity, blood level of medication, metabolizer status or other factors. For example, knowing whether a patient receiving paroxetine is a poor metabolizer of CYP2D6 might be very helpful, as blood levels of paroxetine are likely to be much higher for a poor metabolizer than an extensive metabolizer [23,24]. While this can be important clinical information for physicians, it is inherently difficult to calculate clinical sensitivity or specificity of this information. As such, attempting to evaluate the pharmacogenomic tests using traditional dichotomous variables is disingenuous, yielding arbitrary, rigidly defined outcomes that may diffuse the clinical impact of the test.

Interpretation of pharmacogenomic testing differs significantly from most molecular diagnostic testing, making its evaluation unique and rendering the concept of analytic and clinical sensitivities and specificities complex. Clear delineation of these differences is of utmost importance for laboratories, regulatory agencies, healthcare payers, clinicians, basic scientists and most importantly patients who are all poised to benefit from a thoughtful assessment of these concepts as it relates to the evaluation of pharmacogenomic testing.

Supplementary data

To view the supplementary data that accompany this paper please visit the journal website at: www.futuremedicine.com/doi/suppl/10.2217/pme-2017-0094

\section{Acknowledgements}

The authors would like to thank K Brown for her help in preparing this manuscript.

Financial \& competing interests disclosure

At the time of this study, MR Jablonski, N King, Y Wang, JG Winner, LR Watterson, S Gunselman and BM Dechairo were employees of Assurex Health, a Myriad company, and receive salary and stock options. The authors have no other relevant affiliations or financial involvement with any organization or entity with a financial interest in or financial conflict with the subject matter or materials discussed in the manuscript apart from those disclosed.

No writing assistance was utilized in the production of this manuscript. 


\section{Summary points}

- Combinatorial pharmacogenomics tests have become increasingly utilized by healthcare professionals in the treatment of neuropsychiatric conditions in order to determine an effective treatment plan.

- Combinatorial pharmacogenomic testing utilizes an individual's genomic variation to guide medication selection with the goal of decreasing adverse drug events and increasing efficacy.

- The analytical validity was established for a 12-gene combinatorial pharmacogenomic test that generates a weighted combinatorial report to rank medications according to the severity of gene-drug interactions for an individual.

- The range of genomic DNA concentrations that will produce reliable combinatorial pharmacogenomic test results was $2.5-20 \mathrm{ng} / \mu \mathrm{l}$.

- The precision of each gene component included in this pharmacogenomic test was $\geq 99 \%$.

- Alleles determined using this pharmacogenomic test were evaluated relative to reference genotypes and showed an overall concordance of $99.8 \%$.

- Minor allele frequencies in an admixed North American population were similar to the minor allele frequencies reported for a European population (1000 Genomes Project).

- The examined test possesses robust analytical validity across all genes and alleles tested, specifically demonstrating high rates of accuracy, precision, repeatability and reproducibility across multiple platforms.

Ethical conduct of research

All clinical samples were de-identified for this study and no personal health information was used. All additional samples were obtained from external sources under appropriate institutional review board approval.

Open access

This work is licensed under the Attribution-NonCommercial-NoDerivatives 4.0 Unported License. To view a copy of this license, visit http://creativecommons.org/licenses/by-nc-nd/4.0/

\section{References}

Papers of special note have been highlighted as: $\bullet$ of interest; $\bullet \bullet$ of considerable interest

1. Rush AJ, Trivedi MH, Wisniewski SR et al. Acute and longer term outcomes in depressed outpatients requiring one or several treatment steps: a STAR*D report. Am. J. Psychiatry 163(11), 1905-1917 (2006).

2. Pae CU, Wang SM, Lee SY, Lee SJ. Early switch strategy in patients with major depressive disorder. Expert Rev. Neurother. 12(10), 1185-1188 (2012).

3. Conrado DJ, Rogers HL, Zineh I, Pacanowski A. Consistency of drug-drug and gene-drug interaction information in US FDA-approved drug labels. Pharmacogenomics 14, 215-223 (2013).

- Analysis of concordance between clinically relevant drug-drug interactions and analogous gene-drug interactions for CYP2C19, CYP2D6 and CYP2C9 in US FDA-approved drug labeling.

4. US FDA. Celexa package insert (2017). https://www.accessdata.fda.gov/drugsatfda_docs/label/2017/020822s047lbl.pdf

5. Zhou ZW, Chen XW, Sneed KB et al. Clinical association between pharmacogenomics and adverse drug reactions. Drugs 75(6), 589-631 (2015).

6. Grover S, Kukreti R. HLA alleles and hypersensitivity to carbamazepine: an updated systematic review with meta-analysis. Pharmacogenet. Genomics 24(2), 94-112 (2014).

7. Hall-Flavin DK, Winner JG, Allen JD et al. Using a pharmacogenomic algorithm to guide the treatment of depression. Transl. Psychiatry 2, e172 (2012).

8. Altar CA, Carhart JM, Allen JD, Hall-Flavin DK, Dechairo BM, Winner JG. Clinical validity: combinatorial pharmacogenomics predicts antidepressant responses and healthcare utilizations better than single-gene phenotypes. Pharmacogenomics J. 15(5), 443-451 (2015).

-. Evaluation of the clinical validity of combinatorial phramacogenomic testing in a pooled anlaysis of four independent studies.

9. Altar CA, Carhart J, Allen JD, Hall-Flavin D, Winner J, Dechairo B. Clinical utility of combinatorial pharmacogenomics-guided antidepressant therapy: evidence from three clinical studies. Mol. Neuropsychiatry 1(3), 145-155 (2015).

-. Evidence of clinical utility of combinatorial pharmacogenomic testing in two-arm, prospective clinical trials.

10. Winner JG, Carhart JM, Altar CA, Allen JD, Dechairo B. A prospective, randomized, double-blind study assessing the clinical impact of integrated pharmacogenomic testing for major depressive disorder. Discov. Med. 16, 219-227 (2013).

11. Winner JG, Carhart JM, Altar CA et al. Combinatorial pharmacogenomic guidance for psychiatric medications reduces overall pharmacy costs in a 1-year prospective evaluation. Curr. Med. Res. Opin. 31(9), 1633-1643 (2015). 
12. Winner J, Allen JD, Altar CA, Spahic-Mihajlovic A. Psychiatric pharmacogenomics predicts health resource utilization of outpatients with anxiety and depression. Transl. Psychiatry 3, e242 (2013).

13. Bousman CA, Jaksa P, Pantelis C. Systematic evaluation of commercial pharmacogenetic testing in psychiatry: a focus on CYP2D6 and CYP2C19 allele coverage and results reporting. Pharmacogenet. Genomics 27(11), 387-393 (2017).

14. Kalman LV, Agundez J, Appell ML et al. Pharmacogenetic allele nomenclature: international workgroup recommendations for test result reporting. Clin. Pharmacol. Ther. 99(2), 172-185 (2016).

15. Vanwong N, Ngamsamut N, Hongkaew Y et al. Detection of CYP2D6 polymorphism using Luminex xTAG technology in autism spectrum disorder: CYP2D6 activity score and its association with risperidone levels. Drug Metab. Pharmacokinet. 31(2), 156-162 (2016).

16. Pratt VM, Everts RE, Aggarwal P et al. Characterization of 137 genomic DNA reference materials for 28 pharmacogenetic genes: a GeT-RM collaborative project. J. Mol. Diagn. 18(1), 109-123 (2016).

- Characterization of genomic DNA samples for 28 genes commonly genotyped for pharmacogenomics testing by the Centers for Disease Control and Prevention and Coriell Cell Repositories.

17. The Genomes Project C. A global reference for human genetic variation. Nature 526(7571), 68-74 (2015).

- Report on the completed 1000 Genomes Project, which includes reconstructed genomes of 2504 individuals from 26 populations.

18. College of American Pathologists: proficiency testing (2017). http://www.cap.org/web/home/lab/proficiency-testing?_adf.ctrl-state=13 20q3hx7j_4\&_afrLoop=414838385006671\#!\%40\%40\%3F_afrLoop\%3D414838385006671\%26_adf.ctrl-state\%3Dv56xclpb1_4

19. Database of single nucleotide polymorphisms. National Center for Biotechnology Information, National Library of Medicine, Bethesda, MD, USA, dbSNP Build ID: Human Build 146. www.ncbi.nlm.nih.gov/SNP/

20. De Bakker PI, Mcvean G, Sabeti PC et al. A high-resolution HLA and SNP haplotype map for disease association studies in the extended human MHC. Nat. Genet. 38(10), 1166-1172 (2006).

21. Amstutz U, Ross CJ, Castro-Pastrana LI et al. HLA-A 31:01 and HLA-B 15:02 as genetic markers for carbamazepine hypersensitivity in children. Clin. Pharmacol. Ther. 94(1), 142-149 (2013).

22. Hall-Flavin DK, Winner JG, Allen JD et al. Utility of integrated pharmacogenomic testing to support the treatment of major depressive disorder in a psychiatric outpatient setting. Pharmacogenet. Genomics 23(10), 535-548 (2013).

23. Saruwatari J, Nakashima H, Tsuchimine S, Nishimura M, Ogusu N, Yasui-Furukori N. Possible impact of the CYP2D6*10 polymorphism on the nonlinear pharmacokinetic parameter estimates of paroxetine in Japanese patients with major depressive disorders. Pharmacogenomics Pers. Med. 7, 121-127 (2014).

24. Ueda M, Hirokane G, Morita S et al. The impact of CYP2D6 genotypes on the plasma concentration of paroxetine in Japanese psychiatric patients. Prog. Neuropsychopharmacol. Biol. Psychiatry 30(3), 486-491 (2006). 
